\title{
l'aide du f.f.n. dans le département des pyrénées-atlantiques
}

L'équipement des forêts réalisé avec l'aide du Fonds forestier national s'est jusqu'à présent principalement traduit dans le département des Pyrénées-Atlantiques par la construction de voies d'accès et de pénétration dans les principaux massifs boisés communaux et syndicaux soumis au régime forestier. Certaines zones ont bénéficié en priorité de cet équipement; en particulier les forêts de la montagne basque dont la plus importante, celle du Syndicat du Pays de Soule compte 7600 ha environ.

La Montagne souletine située au sud du département des Pyrénées-Atlantiques à la limite de l'Espagne constitue la province orientale du Pays Basque. Cette zone est soumise aux influences atlantiques (la pluviométrie y est très importante et assez bien répartie dans l'année : 1300 à $2000 \mathrm{~mm}$ de pluies par an) et aux effets de foehn, vent chaud et violent soufflant du sud. Les sols sont en général acides et très riches en matière organique (pluviosité et couvert végétal important).

La population essentiellement agricole exploite de petites exploitations dans les vallées. Ces exploitations dont la majorité dlspose de 3 à 10 ha de surface agricole utile en fond de vallée ont une spéculation de base qui est l'élevage de brebis laitières. Le troupeau bovin (" Blonde des Pyrénées ") apparaít comme complémentaire du troupeau ovin : il utilise les mauvais foins et les mauvaises pâtures. A titre d'élevage traditionnel subsiste celui des pottioks, chevaux sauvages autrefois utilisés dans les mines. Le système d'alimentation fait une large place a l'utilisation des paturages de montagne (de mai à septembre). La production se caractérise donc par une utilisation de terrains exploités plus ou moins intensivement au niveau de l'exploitation et une utilisation complémentaire extensive au niveau des pâturages de montagne.

Ces påturages de montagne sont propriétés communales ou syndicales. Le syndicat de Soule (qui dans le canton de Tardets possede $35 \%$ de la surface) représente les vestiges de l'ancien vicomté de Soule constitué en 1020. Ses terrains sont propriété indivise des habitants de 43 communes. Ils sont constitués de bois pour $56 \%$ de la surface et de landes et pâtures pour $44 \%$ de la surface.

Le terroir agricole ainsi formé constitue une zone d'attraction touristique et cynégétique.

A la beauté des sites s'ajoute l'intérêt que peut présenter la possibilité de chasse à la palombe (colombidé migrateur qui, lors de son passage vers le sud, emprunte les cols dans lesquels il passe en nombre) ou que pourrait présenter la chasse au grand gibier.

Condition de la survie rurale, la modernisation de l'agriculture locale est rendue possible par l'utilisation optimale du territoire agricole et par l'intensification due à l'amélioration des techniques de production.

L'utilisation optimale du territoire passe par une délimitation de zones en fonction de leurs potentialités, ces potentialités étant fonction principalement de la pente des terrains. Ainsi les landes seront-elles transformées en påturages si leur pente est inférieure à $30 \%$ (pente au delà de laquelle des engins mécaniques de défrichement ne peuvent plus être utilisés) ; lorsque la pente sera supérieure à $30 \%$, il sera préférable de replanter des essences forestières. Une des conditions de la meilleure utilisation du sol consiste donc en un défrichement ou un boisement des landes et fougeraies actuelles non utilisées. 
L'intensification par l'amélioration des techniques consiste d'une part en une lutte pour l'augmentation des rendements herbagers (suppression du surpâturage existant dans certaines zones par une utilisation rationnelle sectorisée du terrain, enrichissement du sol par le moyen de la fertilisation chimique) et d'autre part en une amélioration des structures de production (sélection des races, techniques de traite, choix de bâtiments appropriés et construction de centres pastoraux modernes).

Ces quelques réflexions sommaires sont la base des plans de développement agricole de cette région. Elles sont d'ailleurs la base du développement rural dans son ensemble. Le développement touristique ne pourra en effet atteindre sa plénitude que lorsqu'il s'introduira dans un milieu agricole adapté et vivant offrant au citadin un cadre naturel agréable équilibré économiquement, car son introduction brutale dans un milieu qui n'aurait pas atteint un stade d'équilibre suffisamment stable cristalliserait une situation à son niveau d'inadaptation. L'arrivée limitée de la vie touristique peut cependant être la source de revenus immédiats permettant et accélérant la mutation agricole. II faudrait donc se limiter à l'exploitation des ressources touristiques de rentabilité rapide et d'influence limitée : les ressources cynégétiques par exemple (location de postes de chasse à la palombe, mise en adjudication de lots aménagés de chasse au grand gibier).

Au stade de la mise en œuvre de l'aménagement introduit par ces quelques réflexions un type d'action possible dans l'immédiat et prioritaire s'est présenté : l'équipement en voie d'accès de toutes natures.

\section{LES ROUTES FORESTIĖRES DANS LA COMMUNE DE LARRAU}

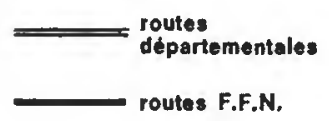

routes flnancées par d'autres moyen

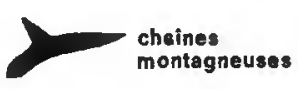

forets communales

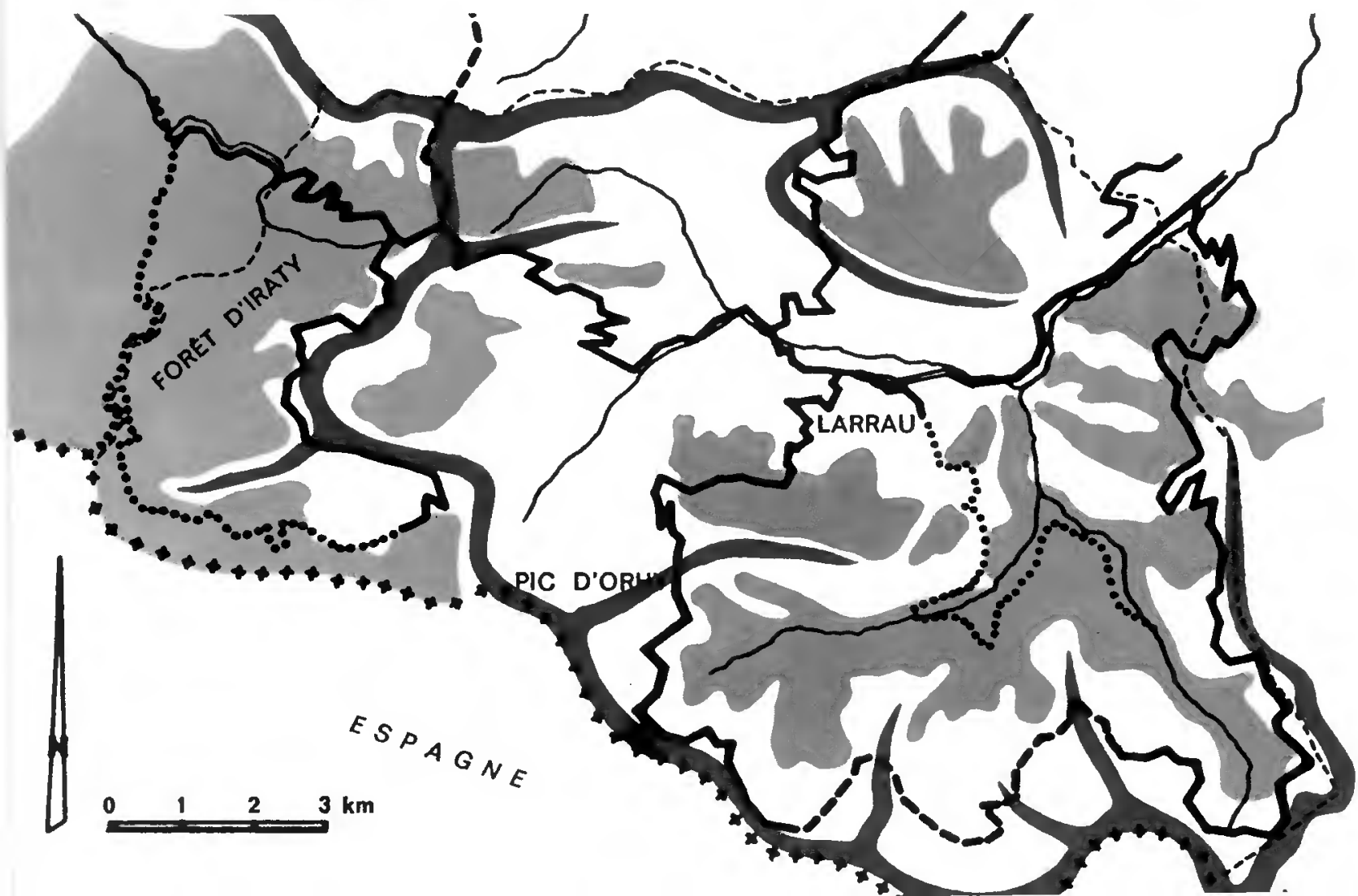




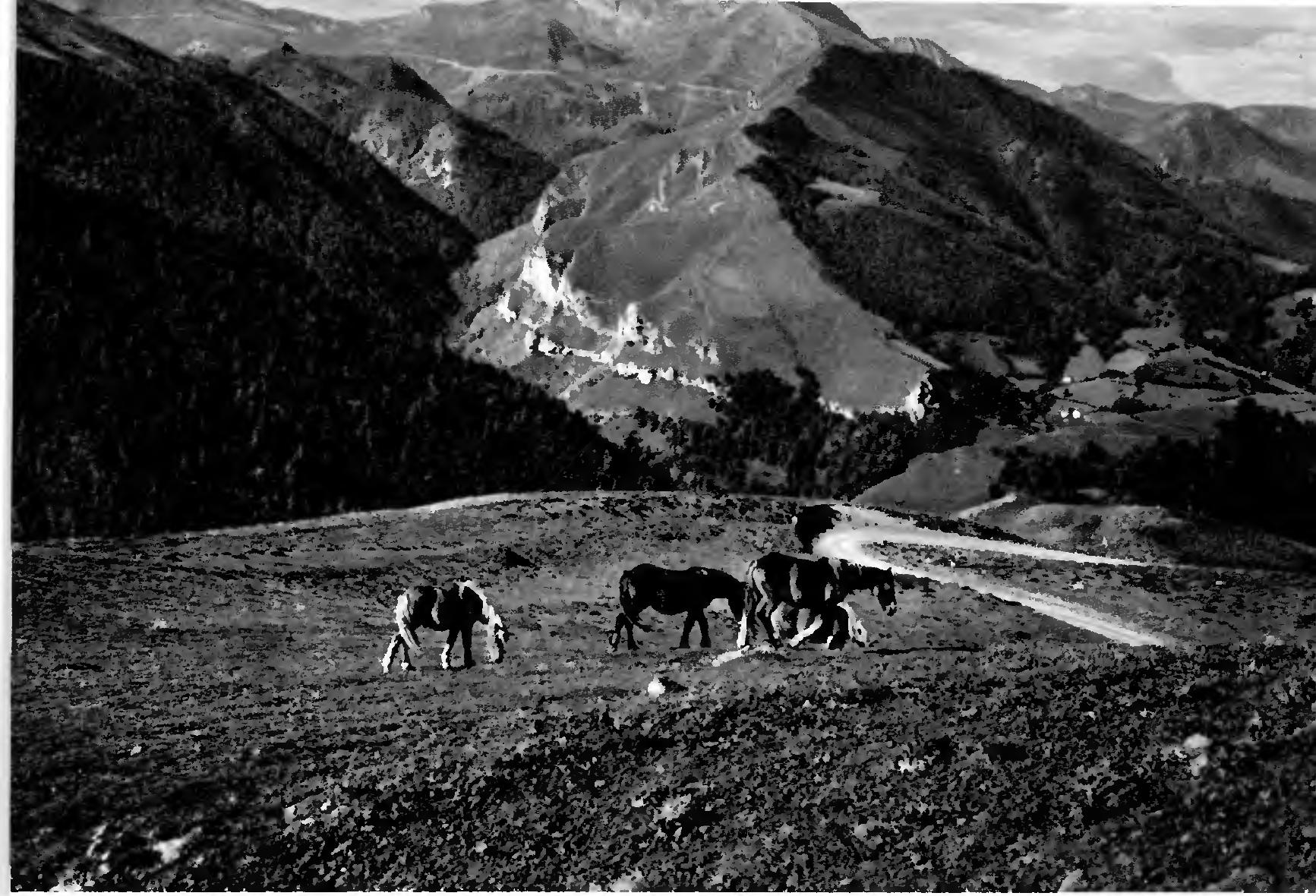

Photo LAzERGES

La desserte des landes et páturages est en effet nécessaire pour permettre d'une part l'accès des engins agricoles nécessaires à la remise en valeur des landes et à la modernisation de la façon culturale des páturages, d'autre part la circulation facile des pasteurs qui sans cela abandonneraient leur activité.

La desserte des forêts est également nécessaire afin d'augmenter le montant des ventes de bois, celui-ci servant à financer la modernisation agricole.

La desserte touristique et cynégétique (circuit de promenades et voies d'accès aux postes de chasse) doit compléter ce réseau pour des raisons identiques.

Il a donc fallu mettre au point un réseau routier efficace et complet au sein duquel les routes d'équipement forestier tiennent une place importante. C'est le syndicat du Pays de Soule qui a en grande partie été le support local de ces travaux d'équipement, l'Etat lui venant en aide au moyen de subventions ou de prêts sur divers chapitres budgétaires ou fonds indépendants (chapitres budgétaires forestiers : améliorations pastorales, investissements de production, prêt pour l'amélioration de la production agricole (forêts) - Fonds forestier national et Fonds européen d'organisation et de garantie agricole.)

De manière générale et pour des raisons écologiques et géographiques, les forêts occupent la zone intermédiaire la plus pentue entre la partie plate du fond de valiée (champs cultivés) et les påturages d'altitude de pente moyenne. Ainsi il peut paraitre logique qu'une route dite de voirie rurale soit prolongée par une route d'équipement réalisée grâce au Fonds forestier, celle-ci étant elle-meme poursuivie par une route pastorale menant ensuite à des cabanes de chasse. 
Ce schéma n'est pas toujours respecté car les séparations entre zones boisées et zones de påturages ne sont pas exactement dues à l'altitude. D'ailleurs une voie pastorale d'accés á une pâture en mauvais état peut devenir à l'avenir une voie forestière, le boisement par semis naturels ou plantations artificielles s'effectuant en cas d'abandon pastoral dans une zone à trop forte pente.

Pour ces raisons et pour celles précédemment exposées voulant que les intérêts économiques divers soient liés, il est extrêmement difficile de distinguer nettement pour chaque projet la part des différents intérêts en cause (forestier, pastoral, cynégétique, divers). Cette distinction est d'ailleurs impossible sur le plan financier, un projet ne pouvant recevoir des aides superposées de différents chapitres budgétaires. L'impossibilité ici décrite conduit à des situations pouvant paraître anormales : une route ouverte grâce à un prêt pour l'amélioration de la production agricole (forêts) sera ensuite goudronnée avec des crédits de voirie pastorale voire même de voirie départementale.

L'équipement routier, qui n'est pas un but en lui-même, a déjà fait augmenter les recettes directes du Syndicat du Pays de Soule qui en 1972 par exemple a pu louer des postes de chasse pour $450000 \mathrm{~F}$ et vendre des bois pour $1420000 \mathrm{~F}$ environ. Dans certaines zones, l'équipement routier et les revenus qu'il rapporte commencent à être suffisants et permettent de passer à la réalisation des équipements conditionnés par eux : construction de centres pastoraux modernes, fumure de pâturage. Cette deuxième phase de l'aménagement doit nous amener à concevoir un pays rural vivant dans lequel le tourisme pourra enfin sintroduire rationnellement, un pays rural dans lequel la forêt participera à l'organisation agricole de maniére vivante.

Le Fonds forestier national par le biais de ses prêts d'équipement œuvre donc, tout en rentabilisant la forêt, à la réalisation pratique d'une politique d'aménagement rural volontaire. Son action ne peut dans l'esprit de ceux qui le mettent en œuvre être séparée de celle-ci. A l'heure de faire le bilan de 25 années d'action, on ne peut que se féliciter d'avoir pu, grâce à lui, relier la politique forestière à une politique d'aménagement globale.

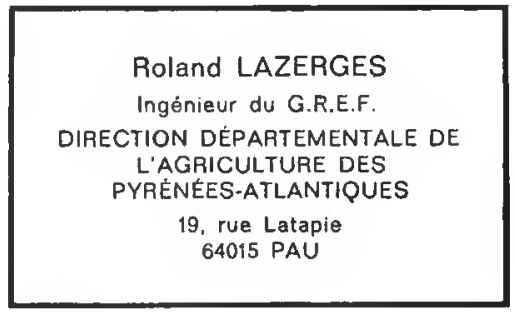

